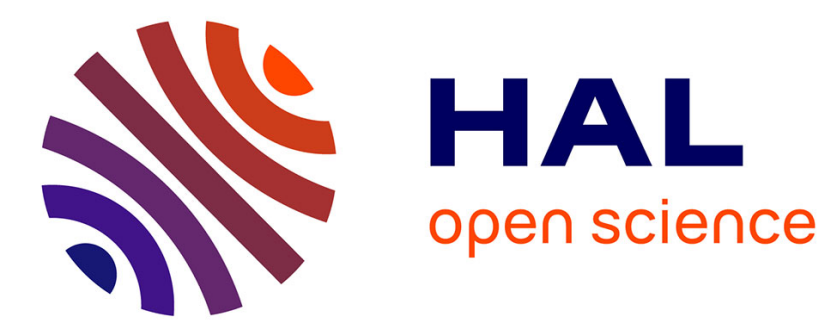

\title{
Le réseau des villes de dimension européenne
}

Denise Pumain, Thérèse Saint-Julien

\section{To cite this version:}

Denise Pumain, Thérèse Saint-Julien. Le réseau des villes de dimension européenne. Hommes et Terres du Nord, 1993, 1, pp.15-25. hal-01524107

\section{HAL Id: hal-01524107 \\ https://hal.science/hal-01524107}

Submitted on 17 May 2017

HAL is a multi-disciplinary open access archive for the deposit and dissemination of scientific research documents, whether they are published or not. The documents may come from teaching and research institutions in France or abroad, or from public or private research centers.
L'archive ouverte pluridisciplinaire HAL, est destinée au dépôt et à la diffusion de documents scientifiques de niveau recherche, publiés ou non, émanant des établissements d'enseignement et de recherche français ou étrangers, des laboratoires publics ou privés. 


\title{
Le réseau des villes de dimension européenne
}

\author{
par Denise PUMAIN et Thérèse SAINT-JULIEN \\ Université de Paris I
}

\begin{abstract}
Résumé
L'ouverture des territoires par la multiplication des liens internationaux s'accompagne d'une redéfinition de la position des grandes villes de chaque pays, dans un réseau urbain qui se structure à l'échelle européenne. Pour esquisser la configuration future de ce réseau, on considère les effets probables de la dimension des villes, de leurs spécialisations fonctionnelles et de leur situation géographique. La typologie obtenue combine un classement hiérarchique et un éventail de spécialisations très diversifié.
\end{abstract}

Mots-clés: Système de villes; Europe; hiérarchie; fonctions urbaines; réseaux.

\section{Introduction \\ Le système des villes européennes: interdépen- dances, dimensions, spécialisations}

L'intégration européenne ne saurait se réduire à ses aspects économiques et politiques, elle est aussi un phénomène d'ordre géographique. L'intégration se traduit dans l'espace géographique par la multiplication et l'intensification des liaisons entre les territoires européens, régions et villes. La construction de nouvelles infrastructures de transport et de communication, les déplacements de personnes et les échanges d'information, la constitution de réseaux plus ou moins formalisés entre les entreprises, les associations et les collectivités locales, se sont récemment accélérés. Il en résulte des interdépendances croissantes entre les territoires, qui deviennent de plus en plus solidaires dans leurs fonctionnements et dans leurs évolutions. En particulier, les grandes villes européennes, qui sont des noeuds essentiels de la vie de relation, se trouvent de plus en plus intégrées dans un ensemble cohérent, elles participent au fonctionnement et au devenir d'un véritable système de villes européen.

Les villes n'étant pas des entreprises, on pourrait penser que les interdépendances qui les lient sont surtout des relations de partage des fonctions et de complémentarité. En fait, on observe qu'au cours des temps historiques, les réseaux urbains nationaux se sont formés dans une compétition permanente des villes pour l'innovation, l'attraction des personnes, des activités et des équipements. Certes, cela s'est produit dans des conditions de vitesse moindre des communications et avec des relations à plus courte portée, mais tous les spécialistes s'accordent à penser que l'on ne peut que retrouver les mêmes processus à l'échelle européenne: les villes s'ajustent aux changements techniques et socio-économiques et les produisent, dans une atmosphère où les rivalités s'expriment plus souvent que la volonté de coopération.

Pour diverses raisons touchant à la gestion territoriale, on peut se demander quels sont les noeuds qui ont un rôle important dans un tel réseau de villes, quels sont les lieux où se joue la compétition urbaine actuelle et

\begin{abstract}
The system of cities with an European size

The position of major cities in their national states has been redefined owing to the multiplication of international communications. Thus major cities are now situated in a European-wide urban network. By considering the probable effects of the physical dimension, the functional specificity and the geographical location of cities, we attempt to sketch out the form that this future network will take. The resulting typology combines a hierarchical ordering and a rather diverse set of specializations.
\end{abstract}

Key-words: Urban system; Europe; hierarchy; urban functions; networks.

à venir. De nombreux travaux déjà publiés ou en cours se sont penchés sur l'identification de villes de dimension "européenne», et ont tenté d'établir des listes de critères significatifs. Plusieurs ont publié des «tableaux d'honneur " énumérant et classant les villes selon leur position dans une hiérarchie: par exemple la typologie établie par R. Brunet (1989) d'après une batterie de 16 indicateurs ou de cinq critères importants, ou encore l'évaluation proposée par P. Cheshire et D. Hay (1989) de l'acuité des problèmes économiques dans les villes européennes.

Tous ces travaux n'ont pas évité cependant certains écueils qui tiennent à la difficulté de l'exercice. Le premier consiste dans l'absence d'une définition de la ville qui soit unique et homogène pour l'ensemble des pays d'Europe. Lorsqu'il s'agit d'évaluer l'importance des villes, l'entité pertinente n'est pas l'unité administrative, mais au moins l'agglomération, éventuellement une région urbaine fonctionnelle ou un bassin d'emploi, à condition que leur délimitation soit assez rigoureuse. La carte des grandes agglomérations européennes de plus de 200000 habitants donne une première approximation du maillage urbain fondamental parmi lequel une sélection de têtes de réseau pourrait être opérée (figure 1).

Une seconde difficulté surgit si l'on se contente de mesurer la dimension des villes par le seul dénombrement de la population de l'agglomération. En particulier, les villes très spécialisées, dans une seule fonction ou presque (villes de la production industrielle, ports maritimes, ou même villes à fonction exclusivement universitaire), ne jouent un rôle international vraiment important que pour la branche d'activité dans laquelle elles sont spécialisées; le plus souvent, leur rôle régional est plus faible, à population égale, que celui de villes aux fonctions plus diversifiées. Si dans l'ensemble une grande agg/omération de population est un critère majeur, qui donne aux villes une forte probabilité d'assumer un rôle important dans la compétition internationale, il faut corriger cette première approximation. A taille égale, les villes européennes jouent des rôles extrêmement différents dans les organisations administrati- 


\section{LES AGGLOMERATIONS EUROPEENNES}

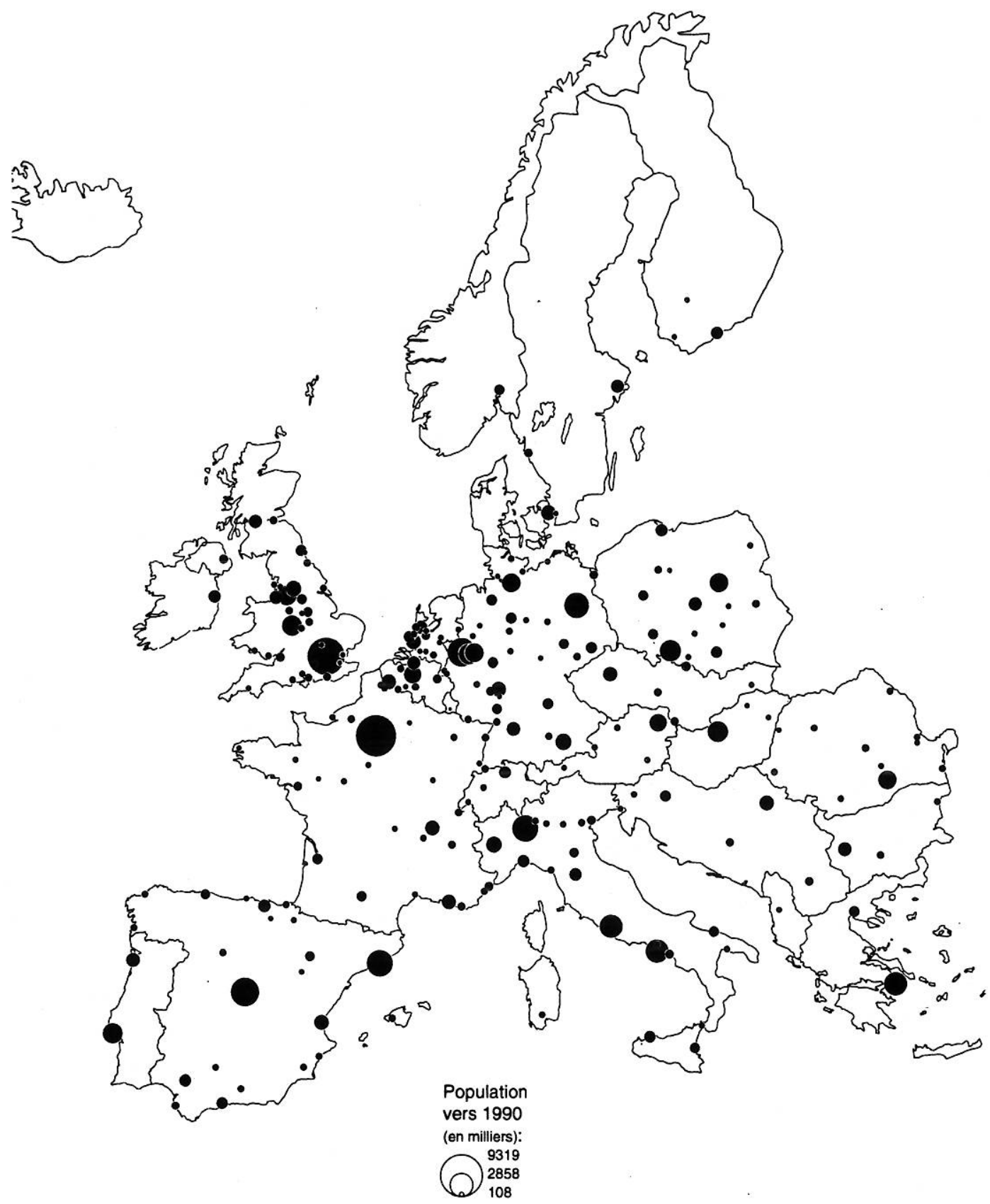

Source: Géopolis

Cartographie: C.Rozenblat, 1993

Figure 1. La population des agglomérations de plus de 200000 habitants.

ves, la production des biens et de la valeur ajoutée, la distribution des services, le rayonnement régional et la diffusion des innovations et du développement.

Une bonne sélection en vue de la préfiguration d'un futur réseau des villes européennes doit donc s'appuyer sur des critères de dimension et sur des critères fonctionnels. Elle doit aussi prendre en compte la situation géographique des villes, en l'évaluant dans une perspective évolutive: toutes choses égales quant au poids démographique et à la diversité des fonctions exercées, une agglomération unique a toute chance d'exercer un rayonnement plus vaste qu'une conurbation polynucléaire; une ville en position centrale dans une région sera plus influente qu'une ville périphérique; une ville bien située à un carrefour de voies de communication (routières, ferrées, aériennes) prendra le pas sur une simple ville étape ou sur une ville située en bout de ligne; quant à la position frontalière, qui s'est souvent traduite jusqu'à présent par une réduction des zones d'influence urbaine, il se pourrait qu'elle évolue plus favorablement, dans la mesure où l'harmonisation des conditions de part et d'autre valoriserait la situation de tête de pont de ces villes.

Un classement rigoureux des villes dans cette perspective exigerait des mesures précises, d'une part de la fonction internationale des villes dans le réseau urbain 
européen, et d'autre part de leur rayonnement réel. De telles mesures n'existent pas encore. Les véritables études expérimentales et comparatives, à partir de matériaux empiriques éprouvés, restent à faire. La Communauté européenne en a lancé plusieurs; en France, après avoir publié le rapport de $\mathrm{R}$. Brunet (1989), la DATAR y consacre l'essentiel de son programme de prospective urbaine, et plusieurs équipes de recherche mènent des travaux dans cette direction; en Europe, le réseau NUREC, qui associe des services statistiques des villes et des institutions de recherche de tous les pays de la Communauté, a été fondé explicitement dans ce but. Plusieurs recherches sont en cours afin de définir une liste pertinente de fonctions internationales (Bonneville et al., 1992).

En revanche, même si les géographes ont depuis longtemps suscité divers travaux pour délimiter des zones d'influence des villes, ces études ne sont pas homogènes d'un pays à l'autre, beaucoup sont trop anciennes, et leur réunion ne permettrait guère que de dresser une carte très provisoire d'un rayonnement régional effectif. Dans la pratique, c'est la diversité des fonctions économiques qui sera utilisée pour compléter cette information lacunaire ou incertaine et pour déterminer indirectement les nuances à apporter à la hiérarchie des poids urbains.

C'est donc en combinant les quatre ensembles de critères: dimension, diversité fonctionnelle, environnement économique local et situation géographique, que l'on peut tenter d'identifier une liste de villes susceptibles de jouer en Europe un rôle efficace dans un réseau d'échanges et d'encadrement territorial. L'identification de ces villes doit nécessairement combiner une position élevée dans une hiérarchie fonctionnelle et une situation géographique centrale. Il est probable que l'on n'aboutisse pas à un classement unique mais à définir les positions de certaines villes dans plusieurs compétitions correspondant à des rôles urbains différents. L'objectif est à terme d'apporter des réponses claires aux questions que posent tous les observateurs: I'union économique et monétaire va-t-elle accentuer ou réduire les disparités entre les villes et donc les régions d'Europe? Certaines structures urbaines sont-elles plus efficaces que d'autres pour affronter cette évolution? Faut-il privilégier le souci de la compétitivité des métropoles ou celui de l'équité en faveur des villes petites et moyennes? Dans quelle mesure les aménagements et les équipements urbains peuvent-ils modifier la position des villes dans la compétition pour la modernisation et l'amélioration de la qualité de la vie des habitants?

Les sources d'information disponibles et comparables ont limité nos investigations aux villes de la Communauté Européenne, auxquelles on a ajouté celles de la Suisse et de l'Autriche. Il va de soi qu'avec l'ouverture des frontières à l'Est, des classifications ultérieures devraient aussi inclure les villes d'Europe centrale, pour lesquelles jusqu'ici seule la population a pu être déterminée de façon comparable (Moriconi, 1993).

\section{Evolution probable de la hiérarchie des villes}

La position hiérarchique est un critère incontournable pour définir la place des villes dans la compétition européenne, à la fois parce qu'elle recouvre une multiplicité d'indicateurs de l'importance des villes, et parce qu'elle joue un rôle majeur dans l'évolution contempo- raine, du fait de l'attraction des grandes villes sur les activités les plus prestigieuses et des tendances à la métropolisation.

Dans le territoire européen, définir une hiérarchie des villes ne signifie pas uniquement identifier des relations de commandement et de dépendance. La hiérarchie se manifeste d'abord comme une différence de niveau des équipements et des fonctions en termes de rayonnement, de portée géographique. Elle se traduit aussi par une inégale complexité et variété des activités et des sociétés urbaines, qui entraînent de très grandes différences dans la dimension et l'influence des villes.

Cette hiérarchie est douée d'une très grande force d'inertie. La dimension des villes définie par leur nombre d'habitants est un indicateur robuste et synthétique des effets accumulés de l'attraction des villes sur la longue durée (fig. 1 et tabl. 1). Il est vain d'imaginer qu'une ville quelconque puisse supplanter Paris et Londres à la tête du réseau urbain européen, même dans une perspective d'un siècle! De même, la réunion de deux villes voisines de plus de 300000 habitants ne saurait donner l'équivalent d'une agglomération de 600000 habitants, même après plusieurs décennies d'étroites interactions. Ainsi, la conurbation de la Ruhr toute entière rassemble plus de 10 millions d'habitants dans un tissu urbain bâti en continuité, mais cet ensemble est encore en fait structuré autour de plusieurs noyaux (Düsseldorf, Essen-Dortmund-Duisbourg) qu'il faut considérer comme des agglomérations fonctionnellement distinctes. D'ailleurs, la conurbation de la Ruhr, en dehors du nombre d'habitants, ne dépasse jamais Paris et Londres pour n'importe quel autre indicateur hiérarchique.

Quel est le degré de liberté des sociétés européennes face à cette forme d'organisation de la hiérarchie des villes? Certains soulignent que l'évolution contemporaine tend à accentuer les inégalités en termes de poids et de chances de développement: c'est la tendance à la métropolisation, renforcée par l'ouverture internationale, l'accès aux transports rapides et la médiatisation. D'autres insistent au contraire sur les possibilités offertes par les nouvelles formes de communication pour atténuer les inégalités hiérarchiques et favoriser le développement "réticulaire».

\section{La métropolisation}

La hiérarchie des villes ne se mesure pas qu'en termes d'inégalités de poids démographique et économique, elle introduit aussi des différences qualitatives. Dans un système hiérarchisé de lieux centraux, les grandes villes concentrent les activités de service les plus rares, celles dont la portée géographique est la plus importante. Elles en retirent des ressources plus élevées et une société plus diversifiée. Un nouveau processus est venu renforcer les spécificités fonctionnelles des métropoles. En s'amplifiant, la tendance générale à la dissociation géographique des fonctions, au sein d'une même entreprise, selon le degré de travail qualifié incorporé, a renforcé le pouvoir socio-économique des plus grandes villes. Avec leurs bonnes conditions d'accessibilité, la diversité qu'apportent d'importants gisements de main-d'œuvre très qualifiée, et tous les avantages d'agglomération liés à la taille, elles apparaissent mieux placées pour attirer les activités nouvelles. Les activit’́s tertiaires et industrielles en forte croissance, tels les services aux entreprises, ou encore les industries spatiales et aéronautiques, sont de bons exemples de la mise en 
Tableau 1. Les agglomérations européennes (1) de plus de 200000 habitants.

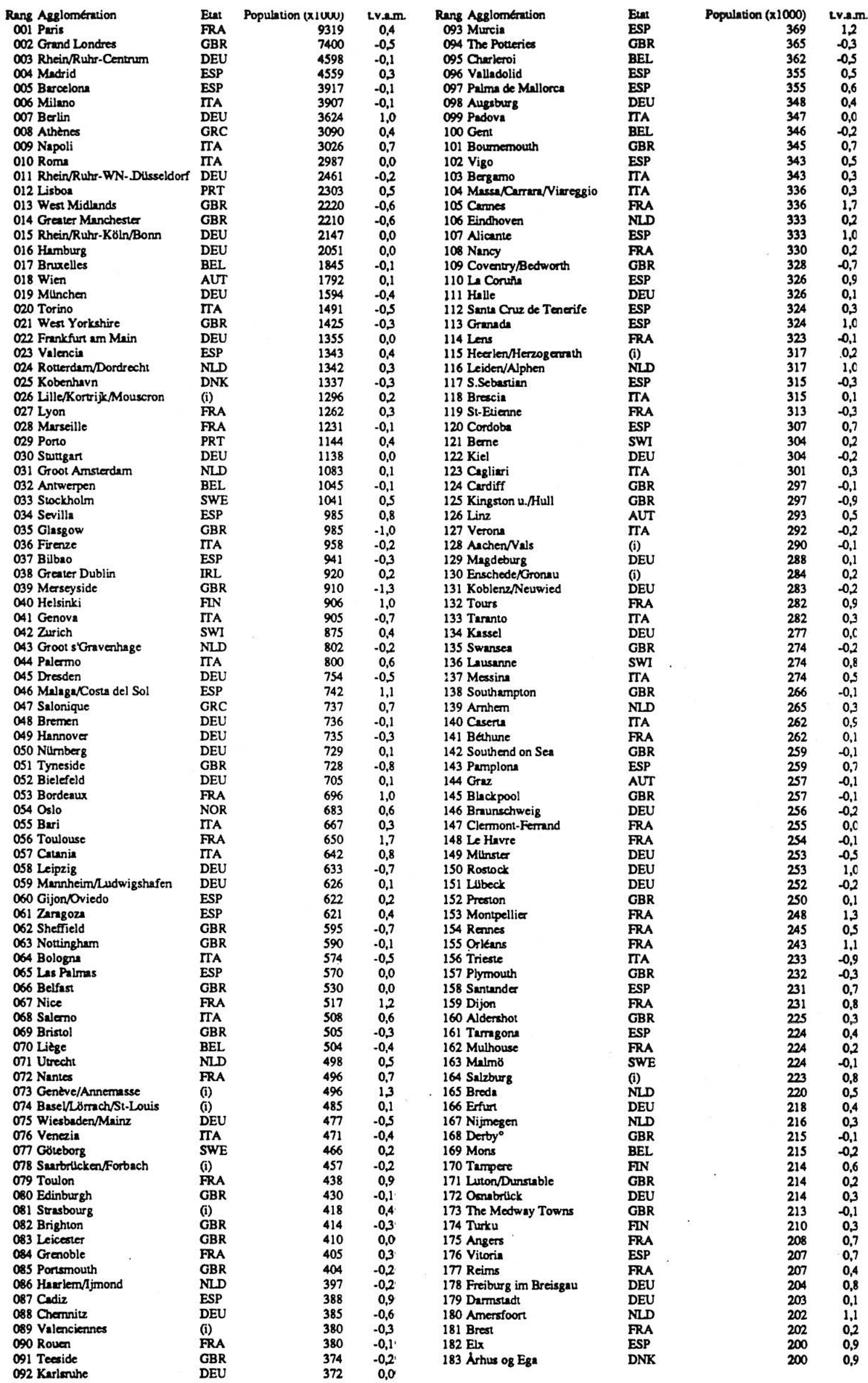

(i) agglomération internationale

t.v.a.m. taux de variation annuel moyen en \% (1980-90)

Source: Geopolis, F. Moriconi-Ebrard

(1) CEE + Autriche, Suisse et Scandinavie 
œuvre de cette forme de division spatiale du travail, qui renforce les spécificités fonctionnelles métropolitaines.

En effet, autant que par les réseaux matériels ou immatériels, l'interconnexion des villes européennes en un système de plus en plus interdépendant s'effectue grâce aux liens développés par les entreprises. L'internationalisation des firmes en est le processus majeur. Cette ouverture a été rendue possible par l'ubiquité croissante des facteurs de production, qui permet une plus grande mobilité des emplois: la CEE (1990) estime que 50\% des emplois sont potentiellement mobiles en 1990, contre $30 \%$ seulement dans les années 1950. La nouvelle division internationale du travail inclut le regroupement dans les grandes villes des emplois qui expriment le pouvoir de décision économique à l'échelon international, c'est un des facteurs essentiels de la métropolisation.

Une enquête sur les facteurs qui influencent la localisation des firmes aujourd'hui dans la CEE (sur 9000 entreprises) révèle quels sont les atouts les plus recherchés (IFO, 1990): des systèmes de communications et de transport rapides et efficaces, la présence d'une force de travail qualifiée, l'accès aux institutions de formation et de recherche, la présence de services aux entreprises de bonne qualité (puisque la tendance est à l'externalisation de ces services par les entreprises), et enfin la qualité de l'environnement social et culturel, incluant les équipements de sport et de loisir. Une enquête parallèle menée en France aboutit à peu près à la même hiérarchie des facteurs (MOCI, 1989, Rozenblat, 1991).

Le processus de sélection des meilleures localisations par les entreprises conduit nécessairement à un renforcement de la position des grandes villes, à la métropolisation dans le système urbain européen. En effet, les facteurs sus-mentionnés sont presque tous corrélés avec la taille des villes: qu'il s'agisse de l'accessibilité, de l'importance des cadres, des universités et de la recherche, de la présence des services aux entreprises, non seulement tous ces indicateurs sont corrélés en valeur absolue à la taille des villes, mais aussi en valeur relative, lorsqu'on les rapporte au nombre d'habitants (Pumain, Saint-Julien, 1989b). Ainsi, I'avantage des grandes villes est-il décisif. II le devient plus encore si I'on ajoute, d'après la plupart des enquêtes, que ce n'est pas tant chacun des facteurs de localisation pris isolément qui joue, mais plutôt leur association, leur rencontre en un même lieu (IFO, 1990).

Bien d'autres travaux mentionnent des indices à la fois plus subtils, plus complexes et sans doute plus significatifs en définitive du processus de métropolisation: jusqu'à présent, ces idées n'ont pas été testées systématiquement.

\section{Les fonctions internationales}

Dans le processus de renforcement des hiérarchies urbaines et de formation du système de villes européen, une attention particulière a été dévolue à l'observation. des fonctions internationales des villes. II s'agit d'énumérer les équipements et les fonctions qui sont le plus susceptibles de permettre aux villes de jouer un rôle important dans les échanges internationaux. Une étude pionnière sur le cas de Bâle (Claustre, 1978) avait proposé de distinguer les "villes internationales», investies par des réseaux internationaux qu'elles ne dominent pas, et des «villes mondiales» qui articulent différents réseaux et participent ainsi à la définition et à la diffusion du nouvel ordre socio-économique mondial. Bien que cette utile distinction n'ait guère été reprise par la suite, c'est surtout la seconde acception qui est retenue pour l'étude des fonctions internationales des villes.

Pour J. Labasse (1981), cinq ensembles de critères caractérisent une ville disposant de fonctions internationales: une population inscrite dans les réseaux internationaux d'échanges économiques et intellectuels, des services de niveau international, des équipements d'accueil pour les manifestations internationales, d'actives colonies de résidents étrangers, et une réputation supranationale dans le domaine du tourisme d'affaires ou culturel.

D'autres auteurs sont partis de considérations très larges, s'appuyant à l'origine sur une conception juridique des relations territoriales (Soldatos, 1989). Les critères retenus pour définir une ville "internationale» par le groupe de Montréal sont: une situation géographique d'ouverture sur le monde, l'accueil de facteurs de production étrangers et l'exportation de facteurs de production à l'étranger, la présence d'institutions et d'entreprises internationales ou d'institutions spécialisées dans le domaine des relations internationales, la facilité des communications avec l'étranger, l'existence d'un secteur de services tournés vers l'international, le rayonnement international des medias, l'accueil de congrès mondiaux, une population ethniquement diversifiée. Un réseau de villes plus spécialement concernées par cette ouverture a été fondé pour en approfondir l'analyse (réseau NICE).

Une recherche menée à Lyon (Bonneville, Buisson, Commerçon, Rousier, 1992) a transcrit ces critères en indicateurs mesurables et identifié parmi seize villes européennes seulement trois villes internationales: Francfort, Amsterdam et Genève. Cet exemple montre que le poids des fonctions internationales ne dépend pas exclusivement de la taille des villes, lorsque l'on se place dans un cadre européen et non plus national. Diverses spécialisations, dans des fonctions politiques, financières ou universitaires, sont susceptibles de rehausser le rang des villes à l'international. Ainsi les sièges sociaux des firmes internationales sont-ils plus nombreux à Londres qu'à Paris, à Francfort qu'à Milan. Pour ces localisations stratégiques, la taille des villes ne joue que par la proximité qu'elle suppose à l'égard d'un pouvoir de décision, politique et financier. De même, l'étude lyonnaise a-t-elle accordé beaucoup de poids au développement des services, bancaires en particulier, et surtout aux liaisons aériennes internationales: les trois villes sélectionnées correspondent aux plus gros aéroports européens. De même, un classement qui privilégierait la recherche scientifique incluerait automatiquement en France les plus grandes villes, mais pas nécessairement dans d'autres pays comme l'Allemagne ou le RoyaumeUni (Brunet, 1989).

\section{De la hiérarchie au réseau}

Contre la tendance à la métropolisation, des observations faites pour la plupart en Italie du Nord apportent quelque espoir aux villes petites et moyennes des réseaux. Selon certains, l'évolution vers le renforcement des contrastes de poids entre les villes ne serait pas inéluctable, ce pourrait même ne pas être la tendance dominante de l'avenir du système urbain européen. Une évolution contraire vers un système de villes moins hié- 
rarchisé, utilisant les moyens modernes de communication pour valoriser une plus grande multiplicité de sites, ressort de certaines observations quant au nouveau partage des fonctions dans des réseaux urbains denses et bien connectés comme ceux du Nord de l'Italie (De Matteis, 1985, Emanuel, 1990a et b).

Des systèmes de villes plus décentralisés seraient tout aussi viables que la séculaire organisation en une hiérarchie de lieux centraux, commandée par le regroupement des fonctions de même portée géographique dans la même ville. La diminution des coûts de transport, la diffusion généralisée de l'accès aux systèmes de communication performants, et la gigantesque contraction de l'espace-temps qui caractérise l'environnement des sociétés contemporaines, permettent l'insertion des activités dans des localisations multiples plus également accessibles.

La contradiction n'est qu'apparente si l'on observe que cette organisation "réticulaire» s'établit à l'intérieur d'une région urbaine, plutôt qu'entre les villes les plus distantes d'un vaste territoire. En outre, la décentralisation et la "dé-hiérarchisation " des fonctions porte bien davantage sur les services à la population et les établissements industriels que sur les services rares et le tertiaire supérieur, qui se concentrent encore (Emanuel, 1990b). En conclusion d'une étude récente, S. Illeris (1991) suggère que le type de système urbain qui émerge dans une société fondée sur les services et l'information reste dominé par un ou plusieurs grands centres, capitales nationales ou régionales, qui sont les mieux intégrées dans l'économie globale de l'information. Il ajoute cependant que d'autres villes, moins grandes mais bénéficiant d'une bonne représentation sociale, sont susceptibles d'attirer une grande partie des activités de pointe, de la recherche, du développement et des innovations. Ces «milieux de prestige» sont quelquefois localisés près de la capitale nationale, comme à Londres, mais en sont parfois très éloignés, comme en France.

\section{Une hiérarchie urbaine en Europe?}

Au total, il semble bien que la constatation par un rapport officiel de l'existence de «hiérarchies urbaines imposées par la compétition internationale» (Fabre, 1991, p. 144) reflète ce que les scientifiques estiment devoir être l'évolution la plus probable d'un système urbain confronté à cette «innovation » majeure que représente l'ouverture des frontières, et une nouvelle accélération de la contraction espace-temps. Evolution qui n'est cependant la plus probable qu'en dehors de toute intervention. Favoriser le renforcement de la hiérarchie au nom de la compétitivité économique est un choix politique qui peut être discuté, il impose en tout cas d'agir avec discernement quant à la sélection entre ce qu'il est nécessaire de concentrer pour une plus grande efficacité, et ce qu'il est possible de maintenir dispersé ou de décentraliser au nom d'une plus grande équité territoriale ou d'une préservation de potentiels variés et viables susceptibles de se trouver valorisés dans des futurs pas nécessairement très éloignés.

Un classement totalement ordonné des agglomérations européennes a été proposé par R. Brunet (1989, p. 55), d'après une synthèse des positions des villes sur seize indicateurs. Ce classement est assez satisfaisant, au moins pour les cinq premières classes. Il a le mérite de corriger l'ordre induit par la seule taille des villes, en essayant d'intégrer la dimension internationale de leurs fonctions. II nous semble cependant que cet ordre peut être utilement nuancé en tenant compte d'une part de la nature de ces fonctions, et d'autre part en accordant une attention aussi grande à la spécialisation de l'ensemble des activités de chaque ville.

\section{Les spécialisations fonctionnelles interna- tionales}

La variété des fonctions urbaines dépend très fortement de règles quasi-universelles de localisation des différentes activités économiques. Les activités de service les plus banales, tributaires au quotidien de la proximité de la population, ont la diffusion la plus large; elles fondent la hiérarchie des lieux centraux, mais ne contribuent guère, pour un niveau hiérarchique donné, à la spécialisation de la fonction des villes. A l'opposé, les activités dont les contraintes de localisation sont les plus strictes introduisent dans le réseau urbain les spécificités fonctionnelles les plus extrêmes, et qui souvent excluent d'autres spécialisations. II s'agit d'activités particulièrement dépendantes d'un gisement particulier, comme le sont par exemple les activités liées traditionnellement aux matières premières industrielles ou aux sites portuaires, et aujourd'hui aux sites touristiques, ou encore de situations plus ou moins centrales ou périphériques.

A un moment donné, les activités économiques contribuent donc de manière variée à la formation des spécialisations fonctionnelles urbaines. En outre dans le temps, le degré de concentration et de clispersion de chaque activité évolue. La tendance à une ubiquité croissante, qui est celle des trois dernières décennies, s'est exercée inégalement selon les activités. En Europe, le développement économique, et les processus de substitution d'activités qui l'ont accompagnée, ont engendré un renouvellement de l'assise fonctionnelle des villes, et une modification de la nature des complémentarités interurbaines que ces fonctions impliquent.

La nature et l'intensité de la spécialisation peuvent contribuer à abaisser ou à élever la position des villes dans un classement hiérarchique. Dans la plupart des pays, les plus grands écarts au schéma hiérarchique général sont introduits par les villes très spécialisées, industrielles, et aussi parfois tertiaires, comme dans le cas de villes touristiques. Les villes très industrielles, et surtout les plus grandes, disposent, par rapport à ce que laisserait supposer leur taille, d'un éventail de fonctions de commandement très réduit, d'un niveau de fonction anormalement bas, et d'une aire d'influence directe de très faible extension: par exemple Liège et Charleroi en Belgique, ou encore La Coruna, Valladolid, et Palma de Mallorca en Espagne, et Lens, Douai, Valenciennes ou Saint-Etienne en France.

Toutes choses égales quant à la taille, les villes capitales d'Etat ont en général des fonctions internationales plus diversifiées et qualitativement supérieures. Leur aire d'influence directe est sans commune mesure avec celle des autres métropoles régionales de niveau immédiatement inférieur. Ce mécanisme renvoie cependant davantage à des spécificités nationales des réseaux urbains, comme par exemple leur degré de primatie. En revanche, des villes de plus faible poids général mais spécialisées dans un type d'activité important pour les relations internationales voient leur position relevée. Les activités financières à Zürich, les activités portuaires à Rotterdam, culturelles à Florence élargissent le rayon- 
nement de ces villes en Europe. La position des petites villes spécialisées risque cependant d'être fragile, et cela d'autant plus qu'elle serait liée à un seul type d'activité et donc trop étroitement dépendante de la conjoncture d'une branche.

R. Brunet (1989, p. 76) a tenté de définir des "profils» de ces spécialisations des villes européennes. Ils sont obtenus à partir des écarts entre le classement hiérarchique général et des classements partiels situant les villes sur cinq groupes d'indicateurs: économie et finan-

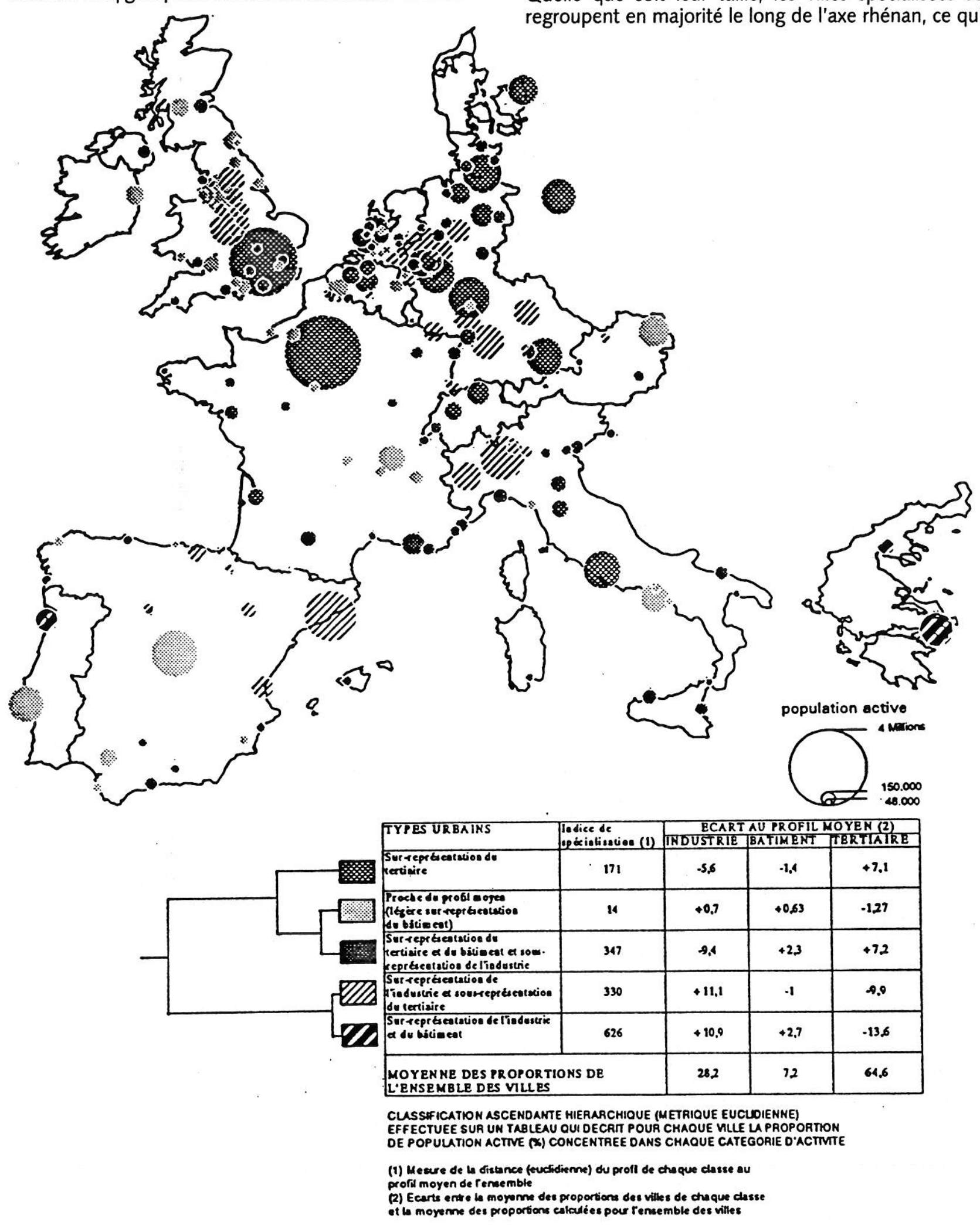

ces, relations internationales, communications, recherche et technologie, et rayonnement culturel. Des villes, souvent de taille moyenne, très rarement capitales d'Etat, apparaissent dans le concert international pour une ou un nombre limité de fonctions. II s'agit d'abord surtout de ports, comme Liverpool, Le Havre, Livourne, Tarente et Brême, mais aussi de villes à fonctions culturelles, telles Nancy, Karlsruhe, Münster, Brunswick, Groningue, Saragosse et Valladolid.

Quelle que soit leur taille, les villes spécialisées se regroupent en majorité le long de l'axe rhénan, ce qui

Source: Recensements de population

Figure 2. Les spécialisations de l'activité économique. 
contribue à définir celui-ci comme l'épine dorsale de l'espace communautaire. En revanche, dans les régions dominées par l'influence d'une métropole, le modèle des villes à fonction internationale spécialisée est moins omniprésent. Si l'on excepte Londres et Paris, qui, du fait du poids et de la diversité de leurs fonctions internationales, ont une position tout à fait dominante et incontestée dans le réseau des villes européennes, les capitales plus périphériques occupent des positions inégales. Certaines affirment, sans partage et sur un espace relativement vaste, leur fonction de capitale régionale à l'échelon européen, (Athènes, Madrid, Barcelone, Milan, Rome, Munich, Berlin, Birmingham, Copenhague, par exemple). D'autres, nombreuses et tout aussi dispersées, ont certes quelques fonctions qui comptent dans la compétition européenne, mais la portée de leur influence est plus limitée: par exemple Lisbonne, Valence, Bordeaux, Naples et Palerme sont dans ce cas. On voit que, dans la perspective de la compétition internationale, certaines villes peuvent apparaître en bonne position pour une fonction donnée, sans qu'elles bénéficient nécessairement de tous les attributs de la "dimension européenne».

Enfin, la portée d'une "fonction internationale» doit être pondérée par l'environnement économique offert par l'ensemble des activités d'une ville (figure 2). La puissance du fait industriel à Birmingham ou à Lille, voire à Leeds-Bradford, invite à imaginer pour elles la possibilité d'exercer un rôle international en ce domaine, que ne leur concédait pas le classement de R. Brunet. De même le développement récent de l'activité culturelle à Glasgow peut faire reconsidérer sa position. Les activités de commerce et de transport peuvent compenser des positions européennes un peu faibles à Rouen, à Palerme, à Padoue, à Southampton par exemple.

\section{Compétition urbaine et situations géogra- phiques}

La situation géographique des villes, leur position dans le système européen et dans les réseaux de communication, sont des éléments importants pour définir leur place dans la compétition interurbaine. Or, la configuration géographique du système urbain européen d'aujourd'hui est très largement héritée, elle ne se modifie que très lentement, à une échelle de temps de l'ordre du siècle, voire davantage. Tout renforcement hiérarchique ou toute innovation fonctionnelle sont donc à relativiser par ces faits de situation.

\section{Centres et périphéries}

Dans l'Europe d'aujourd'hui, l'impression inégalitaire créée par la structure hiérarchique du système urbain se trouve renforcée par l'hétérogénéité de la répartition spatiale de l'urbanisation: près de la moitié des plus grandes villes sont concentrées dans la «Mégalopole» européenne identifiée par E. Juillard (1968) et analysée par R. Brunet (1989). Du centre de l'Angleterre au Nord de l'Italie, sur $1500 \mathrm{~km}$ de long et dans une zone de 100 à 300 km de large, s'échelonnent en arc de cercle des chapelets de villes, insérées dans des réseaux de communication très denses, que n'arrêtent pas les reliefs. La plus forte concentration urbaine du monde, avec 80 millions d'habitants, apparaît bien comme le «centre», le «coeur» de la nouvelle Europe (figure 1).

Au-delà de cette répartition d'ensemble, la concentration métropolitaine caractérise à des degrés divers certains pays, voire certaines des régions d'Europe. La
France n'a pas l'exclusivité de ce mode de concentration. On l'observe dans le sud-est britannique avec Londres, en Castille avec Madrid, en Irlande avec Dublin; il se retrouve à échelle réduite en Aquitaine autour de Toulouse ou Bordeaux, mais aussi en Campanie autour de Naples, et en Bavière dans la mouvance de Munich. La métropole dominante est parfois aussi une ville "primatiale», dont la population dépasse de loin plus de deux fois le poids de la deuxième ville (en France, en Irlande, au Portugal, en Grèce). Même lorsqu'elle n'apparaît pas dans les masses de population, la concentration métropolitaine existe pour les fonctions politiques, ou le pouvoir de décision des entreprises (sièges sociaux): ainsi à Milan, Francfort ou Düsseldorf. Une telle concentration traduit l'effet sur le long terme des économies d'agglomération, mais aussi bien souvent celui de la concentration du pouvoir politique et de décision économique, que renforce la charge symbolique des lieux.

\section{Les spécificités nationales et régionales}

Dans leur étude sur «Espaces et régions en Europe occidentale", Juillard et Nonn (1976) avaient présenté de manière synthétique les spécificités nationales et régionales de l'organisation spatiale des réseaux urbains. Ils montraient en particulier qu'il existe en Europe plusieurs variantes du modèle général, qui lie taille des villes, fonctions de commandement, et aire d'influence. Le modèle "parisien", caractéristique du réseau urbain français, représente le type dans' lequel une ville de très grande taille, domine de manière tout à fait écrasante une aire d'influence très vaste, de 200 à 300 kilomètres de rayon dans le cas de Paris. Dans cette aire, aucune capitale régionale digne de ce nom n'a pu se développer. On peut considérer que le type se retrouve, à des degrés divers, en Grande-Bretagne autour de Londres, au Danemark autour de Copenhague, en Allemagne autour de Munich, en Autriche autour de Vienne, en Italie autour de Milan et de Rome, en Espagne autour de Madrid.

Reprenant la terminologie proposée par Juillard et Nonn, on identifie un type "rhénan", dont le modèle est opposé au précédent: espacement plus réduit des grandes villes, niveaux de fonction moins différenciés (les fonctions les plus rares, voire les plus spécialisées, peuvent être indifféremment exercées par toutes les grandes villes du réseau), aires d'influence plus réduites et beaucoup moins inégales. Ce modèle concerne plus particulièrement les régions rhénanes et flamandes, dont les densités de population sont élevées, et qui n'ont été que faiblement et/ou tardivement soumises à des processus de centralisation économique, et surtout politique, de grande intensité. Entre ces deux types extrêmes, on peut identifier un type «intermédiaire», dont le principe de fonctionnement est très proche du modèle rhénan mais qui, du fait de densités régionales de population beaucoup plus faibles, conduit à une plus large extension des zones d'influence (Italie du nord-est, régions alpines et Lorraine, Levant espagnol, par exemple).

Dans le type "périphérique», seule la métropole régionale assure des services de niveau élevé et étend son influence sur une aire très vaste, dont les marges peuvent être assez mal intégrées (Italie du sud, est de l'Allemagne, sud-ouest de la France). Pour expliquer la présence de ce type, on peut vraisemblablement invoquer des densités de population relativement faibles, 
et des traditions urbaines moins ancrées que dans les régions caractérisées par les types «rhénan» ou «intermédiaire" par exemple, et surtout une distance plus grande aux capitales, qui a protégé les métropoles régionales de leur proximité contraignante.

\section{L'évolution probable des effets de situation}

Compte tenu de la puissance des mécanismes «spontanés" de l'évolution des systèmes de villes, et de la difficulté de définir des objectifs d'ensemble à l'échelle de l'Europe, le réseau des villes est provisoirement condamné à se développer sous le signe de la double contradiction géographique de régions centrales saturées et toujours plus attractives, et de périphéries en croissance démographique, mais laissées un peu à l'écart des formes contemporaines du développement économique.

Les métropoles, en position centrale, insérées dans un milieu très urbanisé, puissamment inter-connectées, particulièrement bien reliées au reste du monde, continueront de sélectionner à leur profit les activités productrices de forte valeur ajoutée, demeurant les pôles majeurs de la diffusion des innovations en Europe. Dans leur voisinage, les villes de taille plus modeste, ne pourront prétendre, dans le meilleur des cas, qu'à un rôle de satellite. Une telle évolution «naturelle» des métropoles centrales, associée à une croissance démographique qui, bien que faible, restera relativement mal contrôlée, y accentuera vraisemblablement les tendances à la saturation des espaces, à la croissance rapide des coûts urbains de fonctionnement, au renforcement des tensions de sociétés urbaines de plus en plus duales. De la réponse donnée à ces contradictions majeures dépendra finalement à terme la dynamique des métropoles centrales.

Dans le même temps, la croissance démographique des centres appartenant aux périphéries du système urbain européen reste rapide. Du sud-ouest au nord-est du continent, du Portugal à la Pologne, en passant par l'Espagne du sud, l'Italie du suci, et l' Europe balkanique, une forte croissance urbarie continuera d'être alimentée par l'exode rural. Au-dela, ies intentions de migration en provenance des régıons situées à l'extérieur, mais au voisinage de cet arc péripherigue (Niaghreb, pays de l'ex-Union Soviétique), se feront plus pressantes. Dans le court terme, les grandes villes de cette périphérie ne disposent pas de solutions analogues à celles qui avaient permis, dans les années 60 , aux villes de la première périphérie (Ouest français, provinces périphériques néerlandaises, ou Bavière, par exemple), de freiner les migrations à grande distance, en fixant régionalement une part importante des populations alors libérées par les mutations de l'agriculture (création rapide d'emplois industriels et tertiaires relativement peu qualifiés), soutenant ainsi une forme de transition socio-économique.

Si rien de comparable ne voit le jour, ces plus grandes villes des régions européennes périphériques, parfois capitales d'Etat, souvent en difficile situation de bout de ligne dans les réseaux des relations intermétropolitaines, risquent d'être confrontées à un afflux très important de population. Elles en seront soit la destination, soit une première étape dans une migration à plus grande distance, vers les métropoles centrales en particulier. Tous ces effets pervers d'interdépendances géographiques trop longtemps ignorées ou mal maîtrisées expliquent l'acuité des problèmes soulevés par l'établissement de nouveaux réseaux de communication en Europe.

Dans la plupart des pays, un groupe de "grandes villes" s'est donc de mieux en mieux individualisé depuis 20 ans. Ce groupe peut être identifié, non seulement par un seuil de taille (qu'on situerait au minimum aux alentours de 500000 habitants), mais aussi par la situation relative de chaque ville dans son réseau urbain national (à population sensiblement égale, Lyon, deuxième ville de France, a moins de poids que Bruxelles, capitale, mais Bordeaux, cinquième ville en France, a plus de poids que Aachen, seizième ville allemande), par la structure des réseaux nationaux (selon qu'ils sont plus ou moins centralisés) et par leur situation géographique dans le réseau des villes européennes (Lille est mieux placée que Valence ou Palerme). L'avance prise par chaque ville dans son propre réseau urbain national sera désormais évaluée à l'aune de la compétition interurbaine européenne, et ajustée à cette échelle.

Un problème particulier est posé par le devenir des villes des ex-zones frontalières. Faut-il attendre des réajustements significatifs dans ces zones? Plusieurs de leurs caractéristiques (interruption des réseaux, hinterlands tronqués...) vont se trouver transformées progressivement et sans doute l'aide de quelques politiques sera nécessaire. Sans attendre, les plus grandes agglomérations ont suscité des aménagements concertés, même en dehors de la CEE (on songe à Bâle et à Genève), et des villes sont associées dans des programmes transfrontaliers de développement régional (par exemple Sar-Lor-Lux).

\section{Situations géographiques et réseaux}

La force des interdépendances qui relient les villes les unes aux autres les intègrent progressivement dans un système urbain européen. Le réseau des villes européennes se constitue par l'élargissement et l'intensification des connexions entre ses noeuds. La preuve la plus visible en est donnée par l'installation des infrastructures matérielles de transport.

Il y a longtemps qu'un réseau de routes et d'autoroutes et de voies ferrées matérialise les échanges en Europe. Mais la révélation des enjeux de la formation d'un réseau de villes européennes, pour le grand public, est venue à l'occasion des discussions occasionnées par la mise en connexion du réseau des Trains à Grande Vitesse. Outre les conflits intra-nationaux quant au tracé des lignes (TGV Nord, TOV Méditerranée en France, TGV Bruxelles-Cologne suscitant un conflit entre Flandre et Wallonie, TGV Paris-Londres refusé au Royaume-Uni), la mise en place d'un réseau de TGV en Europe a entraîné la discussion publique des avantages ou inconvénients de liaisons internationales: ainsi un colloque a-t-il comparé les chances des différentes façons d'utiliser «les couloirs Rhin-Rhône dans l'espace européen » (LET, 1989). Un peu partout, se sont multipliées les études et les rencontres visant à mesurer les effets d'entraînement de cette infrastructure sur le développement économique des villes et l'importance de "l'avantage initial» conféré par un raccordement plus ou moins précoce au réseau (Bieber, 1990). L'extension permanente du réseau autoroutier est une autre occasion de l'intensification des relations et d'expression des concurrences entre villes pour l'accès à l'infrastructure.

Parallèlement, les connexions aériennes se sont multipliées, leur fréquence a augmenté, leur portée s'est élar- 
gie, mais cette intensification de la desserte n'a pas eu le même retentissement pour la prise de conscience d'une interdépendance croissante entre les villes d'Europe. Là encore, du fait des coûts d'investissement et de fonctionnement et de l'importance des immobilisations spatiales que constituent les aéroports internationaux, du fait de leur fréquentation importante par une clientèle de cadres appartenant aux milieux décisionnels des entreprises et répondant aux besoins de contacts "face à face» de la négociation économique, ce sont en général les grandes villes qui se trouvent d'emblée occuper les meilleures accessibilités dans ces réseaux de la desserte aérienne. II s'y ajoute quelques villes devenues des plaques tournantes internationales du fait de leurs fonctions et de leur situation géographique: ainsi, dans une analyse multidimensionnelle de l'accessibilité aérienne des villes européennes, Cauvin, Reymond et Schaub (1989) identifient dans le groupe de tête les villes de Londres, Bruxelles, Amsterdam, Paris, Francfort et Zürich. Les travaux de Cattan (1990) réalisés sur un ensemble plus vaste de grandes villes confirment cette primauté.

$\mathrm{Du}$ fait de la "quatrième révolution logistique» (A. Anderson), l'économie, la culture de masse et la vie sociale sont de plus en plus fondées sur des interactions immatérielles organisées en réseaux. Pour ces relations, la proximité géographique n'est plus un bon prédicteur d'intensité. Et cependant, les télécommunications constituent bien un volet, peu visible mais assez souvent perçu, de la mise en réseau des villes européennes. Certaines villes ont prévu des programmes très lourds d'équipement pour un service offrant aux entreprises une insertion privilégiée dans les réseaux mondiaux: en Europe, Amsterdam, Londres et Cologne sont sur les rangs pour la création de très grands téléports. En France, où le réseau Numeris se développe entre les grandes métropoles, les équipements les plus puissants des "Zones de Télécommunication Avancées" seront localisés à Paris-la-Défense et Toulouse, avec des centres secondaires dans plusieurs technopôles.
Des réseaux d'information réciproque sur les politiques urbaines et les programmes de développement se sont constitués spontanément entre les villes, un peu au hasard des types de villes, de liens établis antérieurement par des jumelages ou selon les familles politiques. Les réseaux de coopération qui se mettent en place sont l'une des expressions du besoin d'information mutuelle qui correspond à la mise en réseau des villes européennes. C'est en effet cette bonne connaissance réciproque qui permet le fonctionnement d'un système de villes régi par la complémentarité, mais aussi par la compétition entre les villes. Néanmoins, ces réseaux semblent participer du hasard de l'auto-organisation. Certains comme le réseau Polis se sont donné explicitement pour objectif le développement de solutions pour les transports urbains, d'autres comme Eurocités, ou ROME ont l'ambition d'être des clubs facilitant l'accès de leurs membres à un niveau européen d'information et de capacité d'échanges.

\section{Conclusion: plusieurs dimensions européen- nes pour les villes}

On ne saurait produire un classement unique des villes susceptibles de jouer un rôle important dans les compétitions européennes à venir. Tout ce qui précède $a$ montré qu'il fallait tenir compte au moins de cinq dimensions: la taille démographique de la ville, le niveau de ses fonctions internationales, l'intensité et la nature de ses spécialisations, la qualité de son environnement économique, et enfin sa situation géographique.

Les deux premiers indicateurs ayant été assez bien pris en compte par le classement général de R. Brunet, nous l'avons repris en le simplifiant et croisé avec une classification synthétique, combinant sa typologie des profils des villes, nos propres analyses des activités des villes et de leur situation géographique (tableau 2). Si l'on s'intéresse à des compétitions entre les villes relevant de considérations d'ordre hiérarchique, on devra lire ce tableau en colonne: par exemple jouent dans la même

Tableau 2. Position des villes en Europe.

\begin{tabular}{|c|c|c|c|c|c|c|c|c|c|c|c|}
\hline \multirow{2}{*}{$\begin{array}{l}\text { Bases de lattraction } \\
\text { statue motropolitaln } \\
\begin{array}{l}\text { Motropoles internationales } \\
\text { dominantes }\end{array}\end{array}$} & \multirow{2}{*}{\begin{tabular}{|l|}
$\begin{array}{l}\text { Pouvoir } \\
\text { de } \\
\text { dedision }\end{array}$ \\
London \\
Pario
\end{tabular}} & \multirow{2}{*}{ 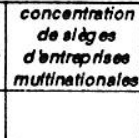 } & \multirow[t]{2}{*}{ 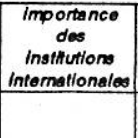 } & \multicolumn{2}{|c|}{$\begin{array}{l}\text { bese dconomique } \\
\text { diversifíe }\end{array}$} & \multirow[t]{2}{*}{$\begin{array}{l}\text { bese } \\
\text { dconomique } \\
\text { spécialis do }\end{array}$} & \multicolumn{2}{|c|}{$\begin{array}{l}\text { Raiblesse de l'kqubemem } \\
\text { tertiaire of du } \\
\text { nivea u deve.ve }\end{array}$} & \multicolumn{2}{|c|}{$\begin{array}{l}\text { modocre accessibilité } \\
\text { asrienne }\end{array}$} & \multirow[t]{2}{*}{$\begin{array}{l}\text { To croissance } \\
19708090 \\
\text { moyenne }\end{array}$} \\
\hline & & & & & & & & & & & \\
\hline $\begin{array}{l}\text { MGropoles Intemationales } \\
\text { id fonction epdelalis }\end{array}$ & & $\begin{array}{l}\text { Ametordam } \\
\text { Dueseldort } \\
\text { Frankfurt } \\
\text { Hamburg } \\
\text { Münchon } \\
\text { Zurlch } \\
\end{array}$ & & & & & & & & & \\
\hline $\begin{array}{l}\text { MGiropoles Internationales } \\
\text { a fonction incomplidte }\end{array}$ & & Bruxelles & $\begin{array}{l}\text { Gendive } \\
\text { Strasbourg }\end{array}$ & $\begin{array}{l}\text { Berlin } \\
\text { Kobenhavn } \\
\text { Wien }\end{array}$ & & & & & & & \\
\hline $\begin{array}{l}\text { MGtropoled roglondes } \\
\text { d fort rayonnement } \\
\text { internatonal }\end{array}$ & & Based & Luxembourg & $\begin{array}{l}\text { Antwerpen } \\
\text { Bremen } \\
\text { Manover } \\
\text { Kóln-Bonn } \\
\text { Lyon } \\
\end{array}$ & $\begin{array}{l}\text { Marselle } \\
\text { Nuremberg } \\
\text { Rotterdam } \\
\text { Stuttgart }\end{array}$ & \begin{tabular}{|l|} 
Berne \\
Bologna \\
Bristol \\
Nice \\
Salzburg \\
\end{tabular} & \begin{tabular}{|l} 
Firenze \\
Mancheot \\
Napoli \\
Roma \\
The Mida \\
\end{tabular} & & & & \\
\hline $\begin{array}{l}\text { Murropolen nationales } \\
\text { on vole d'Internationdisation }\end{array}$ & & & & & & & $\begin{array}{l}\text { Barcelone } \\
\text { Madrid } \\
\text { Miano }\end{array}$ & & & & \\
\hline $\begin{array}{l}\text { MAropoles netionales } \\
\text { leoloce }\end{array}$ & & & & & & & \begin{tabular}{|l} 
Dublin \\
Belfast \\
\end{tabular} & & & & 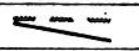 \\
\hline 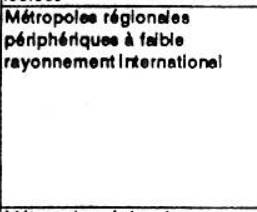 & & $\begin{array}{l}\text { Eindhoven } \\
\text { Cermort- } \\
\text { Ferrand }\end{array}$ & & $\begin{array}{l}\text { Bordeaux } \\
\text { Nantes } \\
\text { Toulouse }\end{array}$ & & $\begin{array}{l}\text { Edinburgh } \\
\text { Innsbruck } \\
\text { Munstor } \\
\text { Southampton }\end{array}$ & \begin{tabular}{|l|} 
Athinai \\
Bilbao \\
Graz \\
Lille \\
Lisboa \\
Palermo \\
Porto \\
Sevilla \\
Thessodor \\
\end{tabular} & $\begin{array}{l}\text { Genova } \\
\text { Glesgow } \\
\text { Unz } \\
\text { Malaga } \\
\text { Torino } \\
\text { Tyneolde } \\
\text { Velencla } \\
\text { Venezla } \\
|k| \\
\end{array}$ & $\begin{array}{l}\text { Mortpollier } \\
\text { Plymouth } \\
\text { Oranada } \\
\text { Orenoble }\end{array}$ & & \\
\hline 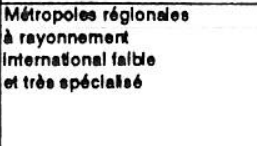 & & & & & & Cardift & \begin{tabular}{|l|} 
Alicante \\
Bari \\
Catana \\
Legge \\
West York \\
Zaragoza
\end{tabular} & & \begin{tabular}{|l|} 
Murde \\
Pamplona \\
Ronnos \\
Sedrt Etionno \\
Velladolld \\
Toulon
\end{tabular} & $\begin{array}{l}\text { Klel } \\
\text { Le Coruna } \\
\text { Lo Havie } \\
\text { San Sebentan } \\
\text { Saltander } \\
\text { Vigo }\end{array}$ & \\
\hline
\end{tabular}


«division» Paris et Londres d'une part, Francfort, Munich, Bruxelles, Milan et Madrid d'autre part. Si la compétition interurbaine concerne des villes d'une même spécialisation fonctionnelle, on devra lire le tableau en ligne et envisager de mettre en concurrence par exemple Lyon, Cologne ou Bristol pour localiser une activité de recherche ou de haute technologie. Le plus souvent, c'est en réalité entre les villes d'une même case du tableau que peut se jouer réellement la concurrence, car il est difficilement concevable que le niveau hiérarchique n'intervienne pas, dans le choix d'une implantation. Parmi les 160 agglomérations des pays de la Communauté Economique Européenne qui avaient au moins 200000 habitants au début des années 80 , une soixantaine peuvent être considérées comme devant jouer un rôle important dans les concurrences interurbaines de demain.

Les études comparatives de l'évolution des villes sur le long terme ont mis en évidence le rôle fondamental de la compétition entre les villes dans la formation et dans l'évolution des réseaux urbains nationaux (Lepetit, 1988, Guérin-Pace, Pumain, 1990). "Cette compétition se joue donc aussi désormais à l'échelle européenne. Les grandes villes rivalisent d'ingéniosité pour accueillir des sièges sociaux, multiplier les fonctions de service, améliorer leur image, étendre leur zone d'influence et accroitre leur puissance et leur séduction " (Fabre, 1991, p. 9). L'ouverture à l'international, comme à l'usage des techniques modernes, conduit les collectivités territoriales, d'intérêt politique ou économique, à se soucier de leur image pour se maintenir ou se hisser au rang de grande métropole européenne. Le message simplificateur du marketing urbain, très inégalement développé d'un pays à l'autre, ne saurait cependant faire oublier les nombreuses dimensions qui interviennent dans la définition de ce rang, et surtout la nécessaire diversité des modèles fonctionnels métropolitains autour desquels continuera de se structurer l'espace européen.

\section{BIBLIOGRAPHIE}

Birber A. 1990: Influence de la grande vitesse sur la restructuration de l'espace européen. UTH 2001, n 13.

Bonneville M., Buisson M.A., Commerçon N., Rousier N. 1992: Villes européennes et internationalisation. Lyon, CNRS, programme Rhône-Alpes en Sciences Humaines, 213 p.

Brunet R., (sous la direction de), 1989: Les villes européennes - Montpellier, GIP RECLUS, rapport pour la DATAR, $85 \mathrm{p}$.

Burtenshaw D., Bateman M., Ashworth G.J.: The European city. A Western perspective. London, David Fulton.

Cattan N., 1991: Une image du réseau des métropoles européennes par le trafic aérien. L'Espace Géographique, $n^{\circ} 2$.

Cattan N., 1992: La mise en réseau des villes européennes. Université Paris I, thèse de doctorat.

Cauvin C., Reymond H., Schaub G., 1989: Accessibilité, temps de séjour et hiérarchie urbaine: l'exemple du réseau aérien pour 53 villes d'Europe. Sistemi Urbani, n 3, 297-324.

CEE 1990: Europe 2000: outlook for the development of the Community's territory. Luxembourg, Communication from the Commission to the Council of the European Parliament, Doc. COM90 544
Champion A.G., Illeris S. 1989: Population redistribution trends in Western Europe: a mosaic of dynamics and crisis, in Hansen J.C., Hebbert $M$. eds., Unfamiliar territory: the reshaping of European geography. Aldershot, Gower.

Cheshire P. Hay D., 1989: Urban Problems in Europe, an economic analysis. London, Unwin Hyman.

Conti S., Spriano G. (a cura) 1990: Effetto città. Turin, Ed. della Fondazione G. Agnelli, $191 \mathrm{p}$.

Dematteis G., 1985: Contro-urbanizzazione e strutture urbane reticolare in: G. Bianchi e I. Magnaghi eds., Sviluppo multiregionale: teorie, metodi, problemi. Milano, Franco Angeli, 121-133.

Dunford M., Kafkalas G. ed. 1992: Cities and Regions in the New Europe. London, Belhaven Press.

Emanuel C., 1990a: Polimorfismo di imprese e di territorio. Rivista di Geografica Italiana, 97, 13-37.

Emanuel C., 1990b: Integrazione urbana e nuove gerarchie di uno spazio regionale: la Padonia centro-occidentale, in R. Innocenti e R. Paloscia eds.: La Riqualificazione delle aree metropolitane, Milano, Franco Angeli, 169-187.

Fabre J., 1991: Le développement des villes françaises de dimension européenne et les réseaux de villes. Paris, Journal Officiel, rapport présenté au Conseil Economique et Social, $n^{\circ} 5,175 \mathrm{p}$.

Guérin-Pace F., Pumain D., 1990: 150 ans de croissance urbaine. Economie et Statistique, $\mathrm{n}^{\circ} 207$.

IFO 1990: An empirical assessment of factors shaping regional competitiveness in problems regions. Bruxelles, rapport pour la CEE, Institut für Wirtschaftforschung.

Juillard E. 1968: L'Europe rhénane. Paris, A. Colin.

Juillard E., Nonn H., 1976: Espaces et régions en Europe Occidentale. Paris, Editions du CNRS, $114 \mathrm{p}$.

Klaassen L.H. 1987: The future of larger European towns. Urban Studies, vol. 24

Labasse J., 1981: Profils de villes européennes à vocation internationale. Cahiers de Géographie du Québec, 25, 66, 403-412.

Lepetit B., 1988: Les villes dans la France moderne (1740-1840). Paris, Albin Michel.

L.E.T. 1989: Les couloirs Rhin-Rhône dans l'espace européen. Lyon, Laboratoire d'Economie des Transports, Actes du colloque, $388 \mathrm{p}$.

M.O.C.I. 1989: Les villes françaises à l'international. $n^{\circ} 895,20$ novembre, 17-24.

Moriconi-Ebrard F., 1993: L'urbanisation du monde d'après Geopolis. Université Paris I, thèse de doctorat.

Parkinson M., Bianchini F., Dawson J., Evans R., Harding A., 1992: Urbanization and the functions of cities in the European Community. Report to Commission of the European Communities (DG XVI), 203 p.

Pumain D., Saint-Julien T., 1989a: Atlas des villes de France. Paris, La documentation Française, $175 \mathrm{p}$.

Pumain D., Saint-Julien T., 1989b: La dynamique d'un système de villes. Sistemi Urbani, 3, 325-338.

Pumain D., Saint-Julien T., Cattan N., Rozenblat C., 1992: Le concept statistique de la ville en Europe. Luxembourg, EUROSTAT.

Rozenblat C., 1991: Les entreprises étrangères dans les villes françaises. Annales de Géographie, $\mathrm{n}^{\circ} 3$.

Rozenblat C., 1992: Le réseau des entreprises multinationales dans le réseau des villes européennes. Université Paris I, thèse de doctorat.

Soldatos P., 1989: La nouvelle génération de villes internationales. Montréal, IEVI. 
